\title{
Implementing Reproducing Kernel Method to Solve Singularly Perturbed Convection-Diffusion Parabolic Problems
}

\author{
Saeid Abbasbandy ${ }^{a}$, Hussein Sahihi ${ }^{b}$ and \\ Tofigh Allahviranloo ${ }^{c}$, \\ ${ }^{a}$ Department of Applied Mathematics, Faculty of Science, Imam Khomeini \\ International University \\ 34149-16818 Qazvin, Iran \\ ${ }^{b}$ Department of Mathematics, Science and Research Branch, Islamic Azad \\ University \\ 14778 Tehran, Iran \\ ${ }^{c}$ Faculty of Engineering and Natural Sciences, Bahcesehir University \\ 34353 Istanbul, Turkey \\ E-mail(corresp.): abbasbandy@ikiu.ac.ir; abbasbandy@yahoo.com \\ E-mail: hu.sahihi@gmail.com \\ E-mail: tofigh.allahviranloo@eng.bau.edu.tr
}

Received February 5, 2020; revised November 19, 2020; accepted November 19, 2020

\begin{abstract}
In the present paper, reproducing kernel method (RKM) is introduced, which is employed to solve singularly perturbed convection-diffusion parabolic problems (SPCDPPs). It is noteworthy to mention that regarding very serve singularities, there are regular boundary layers in SPCDPPs. On the other hand, getting a reliable approximate solution could be difficult due to the layer behavior of SPCDPPs. The strategy developed in our method is dividing the problem region into two regions, so that one of them would contain a boundary layer behavior. For more illustrations of the method, certain linear and nonlinear SPCDPP are solved.
\end{abstract}

Keywords: reproducing kernel method, singularly perturbed parabolic problems, error analysis, boundary layer behavior.

AMS Subject Classification: 35K20; 35K67; 35K55.

Copyright (C) 2021 The Author(s). Published by Vilnius Gediminas Technical University This is an Open Access article distributed under the terms of the Creative Commons Attribution License (http://creativecommons.org/licenses/by/4.0/), which permits unrestricted use, distribution, and reproduction in any medium, provided the original author and source are credited. 


\section{Introduction}

Let us consider the following singularly perturbed convection-diffusion parabolic problem,

$$
\left\{\begin{array}{l}
\mathcal{L}(y(x, t))+\mathcal{N}(y(x, t))=f(x, t) \\
(x, t) \in D \equiv[0,1] \times[0,1] \\
y(0, t)=y(1, t)=0, \quad t \in[0,1] \\
y(x, 0)=y_{0}(x), \quad x \in[0,1]
\end{array}\right.
$$

where $\mathcal{L} y \equiv-\varepsilon \partial_{x}^{2} y(x, t)+p(x, t) \partial_{x} y(x, t)+q(x, t) y(x, t)+\partial_{t} y(x, t)$ and $0<\varepsilon \ll$ 1 , is perturbation parameter and $p(x, t), q(x, t), f(x, t)$ are sufficiently smooth functions such that, $p(x, t) \geq \alpha>0, q(x, t) \geq \beta \geq 0$, and $\mathcal{N}(y(x, t))$ is nonlinear differential operator. Problem (1.1) with the above-mentioned conditions is of a unique solution $y(x, t)$ with boundary layer behavior, in which the boundary layer width is $O\left(\varepsilon \ln \left(\frac{1}{\varepsilon}\right)\right)$ at neighborhood $x=1$, see [22]. SPCDPPs have several applications in various fields of science; for example, the fluid dynamics, electromagnetic field problems, semiconductor device modeling, and meteorological, biological and chemical applications $[6,12,18,20,23,24,30]$. Some applications of the RKM and singularly perturbed problems are introduced in $[14,16,26,28]$. In addition, we can observe applications of the RKM method to solve fractional problems in $[1,2,3,4,5,9,27,29]$.

In this study, the RKM is utilized without the Gram-Schmidt orthogonalization process, which was primarily introduced by Wang et al., [32, 33, 34]. The strategy developed in order to solve these problems with layer behavior is explained in three steps. The first step is splitting the region into two regions, so that one of them would contain a boundary layer behavior; the second step is shifting the layer region to another region, and as the final step, a proper set of collocation points is also required for the boundary layer and regular regions. Since the RKM is a powerful numerical method, if these three steps are properly applied to the problem, this technique will be able to provide an appropriate approximation of the solution, even with severe singularities.

Remark 1. Problem (1.1) with two regions, such that one of the regions contains a boundary layer behavior, therefore $D=D_{1} \cup D_{2}$, where $D_{1} \equiv[0,1-\mu] \times[0,1]$ and $D_{2} \equiv[1-\mu, 1] \times[0,1]$. Since, the RKM does not provide an approximate solution for the right layer region, it is essential to shift region $D_{2}$ to another region, such as $D_{3} \equiv[-1,0] \times[0,1]$. Further details are provided in [34].

\section{Main idea}

\subsection{Preliminaries and notations}

Definition 1. $\mathcal{W}_{2}^{m}[a, b]=\left\{u(x) \mid u^{(m-1)}(x)\right.$ is absolutely continuous,$u^{(m)}(x) \in$ $\left.L^{2}[a, b], u(a)=u(b)=0\right\}$. The inner product and norm in $\mathcal{W}_{2}^{m}[a, b]$ are given 
as follows,

$$
\begin{aligned}
& <u_{1}(x), u_{2}(x)>_{\mathcal{W}_{2}^{m}[a, b]}=\sum_{i=0}^{m-1} u_{1}^{(i)}(a) u_{2}^{(i)}(a)+\int_{a}^{b} u_{1}^{(m)}(x) u_{2}^{(m)}(x) d x, \\
& \|u(x)\|_{\mathcal{W}_{2}^{m}[a, b]}=\sqrt{<u, u>\mathcal{W}_{2}^{m}[a, b]}, \quad u_{1}(x), u_{2}(x) \in \mathcal{W}_{2}^{m}[a, b] .
\end{aligned}
$$

Definition 2. $\mathcal{W}_{2}^{(m, n)}(\mathcal{D})=\mathcal{W}_{2}^{m}[a, b] \otimes \mathcal{W}_{2}^{n}[c, d]=\left\{u(x, t) \mid \frac{\partial^{m+n-2} u(x, t)}{\partial x^{m-1} \partial t^{n-1}}\right.$ is completely continuous in $\left.\mathcal{D}, \frac{\partial^{m+n} u(x, t)}{\partial x^{m} \partial t^{n}} \in L^{2}(\mathcal{D}), u(a, t)=u(b, t)=u(x, c)=0\right\}$.

The inner product and norm in $\mathcal{W}_{2}^{(m, n)}(\mathcal{D})$ are given as follows,

$$
\begin{aligned}
& <u_{1}(x, t), u_{2}(x, t)>_{\mathcal{W}_{2}^{(m, n)}(\mathcal{D})}=\sum_{i=0}^{m-1} \int_{c}^{d}\left[\frac{\partial^{n}}{\partial t^{n}} \frac{\partial^{i}}{\partial x^{i}} u_{1}(a, t) \frac{\partial^{n}}{\partial t^{n}} \frac{\partial^{i}}{\partial x^{i}} u_{2}(a, t)\right] d t \\
& \quad+\sum_{j=0}^{n-1}\left\langle\frac{\partial^{j}}{\partial t^{j}} u_{1}(x, c), \frac{\partial^{j}}{\partial t^{j}} u_{2}(x, c)\right\rangle_{\mathcal{W}_{2}^{m}[a, b]} \\
& \quad+\int_{a}^{b} \int_{c}^{d} \frac{\partial^{m}}{\partial x^{m}} \frac{\partial^{n}}{\partial t^{n}} u_{1}(x, t) \frac{\partial^{m}}{\partial x^{m}} \frac{\partial^{n}}{\partial t^{n}} u_{2}(x, t) d x d t \\
& \|u(x, t)\|_{\mathcal{W}_{2}^{(m, n)}(\mathcal{D})}=\sqrt{\left\langle u, u>_{\mathcal{W}_{2}^{(m, n)}(\mathcal{D})}\right.}, \quad u_{1}(x, t), u_{2}(x, t) \in \mathcal{W}_{2}^{(m, n)}(\mathcal{D}) .
\end{aligned}
$$

Remark 2. Reproducing kernel for spaces $\mathcal{W}_{2}^{3}[0,1-\mu], \mathcal{W}_{2}^{2}[0,1]$ and $\mathcal{W}_{2}^{3}[-1,0]$ are given as follow,

$$
\begin{aligned}
& \dot{\mathcal{R}}_{\eta}(x)=\left\{\begin{array}{ll}
\dot{\mathcal{R}}(x, \eta), & x \leq \eta, \\
\dot{\mathcal{R}}(\eta, x), & \eta>x,
\end{array} \quad \dot{\mathcal{R}}(x, \eta)=\frac{x^{5}}{120}-\frac{\eta x^{4}}{24}+\frac{\eta^{2} x^{3}}{12}+\frac{\eta^{2} x^{2}}{4}+\eta x,\right. \\
& \dot{\mathcal{R}}_{\xi}(t)=\left\{\begin{array}{ll}
\dot{\mathcal{R}}(t, \xi), & t \leq \xi, \\
\dot{\mathcal{R}}(\xi, t), & \xi>t,
\end{array} \quad \stackrel{\mathcal{R}}{ }(t, \xi)=-\frac{t^{3}}{6}+\frac{\xi t^{2}}{2}+\xi t,\right. \\
& \ddot{\mathcal{R}}_{\eta}(x)=\left\{\begin{array}{ll}
\ddot{\mathcal{R}}(x, \eta), & x \leq \eta, \\
\ddot{\mathcal{R}}(\eta, x), & \eta>x,
\end{array} \quad \ddot{\mathcal{R}}(x, \eta)=-\frac{\eta^{5}}{120}-\frac{\eta^{5} x^{5}}{18720}-\frac{\eta^{2} x^{5}}{468}-\frac{\eta x^{5}}{96}\right. \\
&-\frac{\eta x^{4}}{24}+\frac{\eta^{2} x^{3}}{12}-\frac{\eta^{5} x^{2}}{468}+\frac{97 \eta^{2} x^{2}}{234}+\frac{\eta x^{2}}{3}-\frac{\eta^{5} x}{96}+\frac{\eta^{2} x}{3}+\frac{29 \eta x}{96} .
\end{aligned}
$$

\subsubsection{Reproducing kernel spaces for regular region}

$$
\mathbf{D}_{\mathbf{1}} \equiv[\mathbf{0}, \mathbf{1}-\mu] \times[\mathbf{0}, \mathbf{1}]
$$

Consider reproducing kernel spaces $\mathcal{W}_{2}^{(3,2)}\left(D_{1}\right)$ and $\mathcal{W}_{2}^{(1,1)}\left(D_{1}\right)$ where their reproducing kernels are given as $\dot{\mathcal{K}}_{\eta, \xi}(x, t)=\dot{\mathcal{R}}_{\eta}(x) \dot{\mathcal{R}}_{\xi}(t)$ and $\dot{k}_{\eta, \xi}(x, t)=$ $\dot{r}_{\eta}(x) \stackrel{\circ}{r}_{\xi}(t)$, respectively.

\subsubsection{Reproducing kernel spaces for shifted boundary-layer region $\mathbf{D}_{\mathbf{3}} \equiv[-\mathbf{1}, \mathbf{0}] \times[\mathbf{0}, \mathbf{1}]$}

Consider reproducing kernel spaces $\mathcal{W}_{2}^{(3,2)}\left(D_{3}\right)$ and $\mathcal{W}_{2}^{(1,1)}\left(D_{3}\right)$ where their reproducing kernels are given as, $\ddot{\mathcal{K}}_{\eta, \xi}(x, t)=\ddot{\mathcal{R}}_{\eta}(x) \stackrel{\mathcal{R}}{\xi}_{\xi}(t)$ and $\ddot{k}_{\eta, \xi}(x, t)=$ $\ddot{r}_{\eta}(x) \stackrel{\circ}{\xi}_{\xi}(t)$, respectively. See [11]. 


\subsection{Reproducing kernel method}

Consider $\mathcal{L}: \mathcal{W}_{2}^{(3,2)}\left(D_{1}\right) \longrightarrow \mathcal{W}_{2}^{(1,1)}\left(D_{1}\right)$ for the regular region in equation (1.1) where $\mathcal{L}$ is a invertible bounded linear differential operator, and $\mathcal{N}(y(x, t))$ is a continuous nonlinear differential operator, and $y(x, t)$ is an unknown function. The functions $\dot{\mathcal{K}}_{\eta, \xi}(x, t)$ and $\dot{k}_{\eta, \xi}(x, t)$ are reproducing kernels of $\mathcal{W}_{2}^{(3,2)}\left(D_{1}\right)$ and $\mathcal{W}_{2}^{(1,1)}\left(D_{1}\right)$ respectively. Now we choose a countable dense points $\left\{\left(x_{i}, t_{j}\right)\right\}_{i, j=1}^{\infty}$ on the region $D_{1}$ and define,

$$
\phi_{i j}(x, t)=\left.\dot{k}_{\eta, \xi}(x, t)\right|_{(\eta, \xi)=\left(x_{i}, t_{j}\right)}, \quad \psi_{i j}(x, t)=\mathcal{L}^{*} \phi_{i j}(x, t),
$$

where $\mathcal{L}^{*}$ is adjoint operator of $\mathcal{L}$. It is clear that,

$$
\psi_{i j}(x, t)=\left.\mathcal{L}_{\eta, \xi} \dot{\mathcal{K}}_{\eta, \xi}(x, t)\right|_{(\eta, \xi)=\left(x_{i}, t_{j}\right)}, \quad \varphi_{i j}(x, t)=\left.\dot{\mathcal{K}}_{\eta, \xi}(x, t)\right|_{(\eta, \xi)=\left(x_{i}, t_{j}\right)}
$$

are complete functional systems for reproducing kernel space $\mathcal{W}_{2}^{(3,2)}\left(D_{1}\right)$ where $i, j=1,2, \ldots,[32,33,34]$.

Theorem 1. Suppose that the inverse of the linear operator $\mathcal{L}$ exists. The exact solution of the equation (1.1) can be represented as,

$$
y(x, t)=\sum_{\ell=1}^{\infty} \varrho_{\ell} \varphi_{\ell}(x, t),
$$

where $\left\{\left(x_{i}, t_{j}\right)\right\}_{i, j=1}^{\infty}$ are countable dense sequence of points on the $D_{1}$ and $\varrho_{\ell}$ are the unknown coefficients that must be determined and $\ell \equiv i, j$.

Proof. See [34].

Therefore by truncating the series (2.1), we can provide an approximate solution for the SPCDPP (1.1)

$$
\dot{y}_{\dot{n}, n}(x, t)=\sum_{\ell=1}^{\dot{n}} \varrho_{\ell, \dot{n}, n} \varphi_{\ell}(x, t), \quad n=1,2, \ldots,
$$

where $n$ is the number of iteration for nonlinear term $\mathcal{N}(y(x, t))$, and $n$ must be sufficiently large. $\dot{n}$ is the number of collocation points to apply the present RKM in the regular region $D_{1}$ and $\dot{n}_{1} \times \dot{n}_{2}=\dot{n}$. $\ell \equiv i, j$, where $i=1,2, \ldots, \dot{n}_{1}$ and $j=1,2, \ldots, \dot{n}_{2}$. Based on the general technique for nonlinear problem, it is clear that the nonlinear problem (1.1) turns to a sequence of iterations to solve a linear problem in the form, $\mathcal{L}\left(\dot{y}_{\dot{n}, n}(x, t)\right)=\mathcal{N}\left(\dot{y}_{\dot{n}, n-1}(x, t)\right)+f(x, t)$. For $n=1$ we choose the initial function $\dot{y}_{\dot{n}, 0}(x, t)$ such that satisfies in the regular region conditions and for each iteration $n=2,3, \ldots$ we obtain $\mathcal{N}\left(\dot{y}_{\dot{n}, n-1}(x, t)\right)$. Now we determine the unknown coefficients $\varrho_{\ell, \dot{n}, n}$ by using the fundamental concepts of the Galerkin method. Consider the following equation

$$
R_{\dot{n}}(x, t)=\mathcal{L}\left(\dot{y}_{\dot{n}, n}(x, t)\right)-\mathcal{N}\left(\dot{y}_{\dot{n}, n-1}(x, t)\right)-f(x, t),
$$

such that $\left\langle R_{\dot{n}}(x, t), \varphi_{\ell}(x, t)\right\rangle_{\mathcal{W}_{2}^{(3,2)}\left(D_{1}\right)}=0$ for $\ell \equiv i, j$ where $i=1,2, \ldots, \dot{n}_{1}$ and $j=1,2, \ldots, \dot{n}_{2}$ and $\dot{n}_{1} \times \dot{n}_{2}=\dot{n}$. Using equations (2.2) and (2.3) it is 
easy to see that we have the following system of algebraic equations to obtain coefficients $\varrho_{\ell, \dot{n}, n}$ :

$$
\begin{aligned}
& \left.\sum_{\ell=1}^{\dot{n}} \varrho_{\ell, \dot{n}, n} \mathcal{L} \varphi_{\ell}(x, t)\right|_{(x, t)=\left(x_{i}, t_{j}\right)}=\left.\mathcal{N}\left(\dot{y}_{\dot{n}, n-1}(x, t)\right)\right|_{(x, t)=\left(x_{i}, t_{j}\right)}+f\left(x_{i}, t_{j}\right), \\
& n=1,2, \ldots, \quad i=1,2, \ldots, \dot{n}_{1} \quad j=1,2, \ldots, \dot{n}_{2} \quad \dot{n}_{1} \times \dot{n}_{2}=\dot{n} .
\end{aligned}
$$

\subsection{Implementing RKM for SPCDPP}

Consider the regular region $(x, t) \in D_{1} \equiv[0,1-\mu] \times[0,1]$

$$
\left\{\begin{array}{l}
-\varepsilon \partial_{x}^{2} y(x, t)+p(x, t) \partial_{x} y(x, t)+q(x, t) y(x, t)+\partial_{t} y(x, t) \\
=\mathcal{N}(y(x, t))+f(x, t), \quad(x, t) \in D_{1} \\
y(0, t)=0, \quad t \in[0,1] \\
y(x, 0)=y_{0}(x), \quad x \in[0,1-\mu]
\end{array}\right.
$$

Now homogenize the equation (2.5) and solve it by using the RKM in the space $\mathcal{W}_{2}^{(3,2)}\left(D_{1}\right)$. In the right layer region $(x, t) \in D_{2} \equiv[1-\mu, 1] \times[0,1]$ we have

$$
\left\{\begin{array}{l}
-\varepsilon \partial_{x}^{2} y(x, t)+p(x, t) \partial_{x} y(x, t)+q(x, t) y(x, t)+\partial_{t} y(x, t) \\
=\mathcal{N}(y(x, t))+f(x, t), \quad(x, t) \in D_{2} \\
y(1, t)=0, \quad y(1-\mu, t) \text { is known, } \quad t \in[0,1] \\
y(x, 0)=y_{0}(x), \quad x \in[1-\mu, 1] .
\end{array}\right.
$$

Suppose that $x=\mu z+1$ and $y(x, t)=u(z, t)$ such that $\partial_{z} u(z, t)=\mu \partial_{x} y(x, t)$ and $\partial_{z}^{2} u(z, t)=\mu^{2} \partial_{x}^{2} y(x, t)$ therefore the equation (2.6) turns into,

$$
\left\{\begin{array}{l}
\frac{-\varepsilon}{\mu^{2}} \partial_{z}^{2} u(z, t)+\frac{1}{\mu} p(\mu z+1, t) \partial_{z} u(z, t)+q(\mu z+1, t) u(z, t)+\partial_{t} u(z, t) \\
=\mathcal{N}(u(z, t))+f(\mu z+1, t), \quad(z, t) \in D_{3} \equiv[-1,0] \times[0,1], \\
u(0, t)=0, \quad u(-1, t) \text { is known, } \quad t \in[0,1], \\
u(z, 0)=u_{0}(z), \quad z \in[-1,0] .
\end{array}\right.
$$

Now again homogenize the equation (2.7) and solve it by using the RKM in the space $\mathcal{W}_{2}^{(3,2)}\left(D_{3}\right)$.

\subsection{Convergence analysis}

Lemma 1. Suppose $\mathcal{Q}=\left\{\dot{y}_{\dot{n}, n}(x, t) \mid\left\|\dot{y}_{\dot{n}, n}(x, t)\right\|_{\mathcal{W}_{2}^{(3,2)}\left(D_{1}\right)} \leq \stackrel{\circ}{c}\right\}$, then $\mathcal{Q}$ is compact set in space $C\left(D_{1}\right)$, where $\stackrel{\circ}{c}$ is a constant and $n=1,2, \ldots$.

Proof. See $[21,25,32]$.

Theorem 2. Suppose $\dot{y}_{\dot{n}, n}(x, t), y(x, t)$ are the approximate solution in space $\mathcal{W}_{2}^{(3,2)}\left(D_{1}\right)$ and the exact solution for the problem (1.1) respectively, and $\left\{\left(x_{i}, t_{j}\right)\right\}_{i, j=1}^{\infty}$ are dense points on the region $D_{1}$ then $\dot{y}_{\dot{n}, n}(x, t) \longrightarrow y(x, t)$ when $\dot{n} \longrightarrow \infty$. 
Proof. $\quad$ First, we need to show that $\left\|\dot{y}_{\dot{n}, n}(x, t)-y(x, t)\right\|_{\mathcal{W}_{2}^{(3,2)}} \longrightarrow 0$ when $\dot{n} \longrightarrow \infty$ then we prove that $\dot{y}_{\dot{n}, n}(x, t)$ uniformly convergent to $y(x, t)$ using the reproducing properties. See [25].

Corollary 1. Similar to Theorem 2 we have $\partial_{t} \dot{y}_{\dot{n}, n}(x, t) \longrightarrow \partial_{t} y(x, t)$ when, $\dot{n} \rightarrow \infty$.

\section{Error analysis}

\subsection{Preliminaries and notations for the boundary layer region}

Suppose $y(x, t)$ is exact solution for the problem (1.1) with a boundary layer behavior and $\mathcal{Y}_{n}(x, t)$ is approximate solution as following form,

$$
\mathcal{Y}_{N, n}(x, t)= \begin{cases}\dot{y}_{\dot{n}, n}(x, t), & x \in D_{1} \equiv[0,1-\mu] \times[0,1], \\ \ddot{y}_{\ddot{n}, n}(x, t), & x \in D_{2} \equiv[1-\mu, 1] \times[0,1]\end{cases}
$$

where $\dot{y}_{\dot{n}}(x, t)$ and $\ddot{y}_{\ddot{n}}(x, t)$ are approximate solutions that obtained from the present method in the regions $D_{1}$ and $D_{2}$, respectively. Number of collocation points throughout the region $D$ is $N$ and $\dot{n}, \ddot{n}$ are number of collocation points on $D_{1}$ and $D_{2}$, respectively and $N=\dot{n}+\ddot{n}$.

Theorem 3. Suppose the problem (1.1) as linear form which has homogeneous initial-boundary conditions and $f(x, t)$ is sufficiently smooth function such that the following conditions are satisfied

$$
f(1,0)=0, \quad\left|\frac{\partial^{k+m} f(x, t)}{\partial x^{k} \partial t^{m}}\right|_{(x, t)=(0,0)}=0,
$$

where $k+2 m \leq 3$, then we have the following bound for solution of the problem (1.1),

$$
\left|\frac{\partial^{k+m} y(x, t)}{\partial x^{k} \partial t^{m}}\right| \leq C\left(1+\varepsilon^{-k} e^{\frac{-\beta(1-x)}{\varepsilon}}\right), \quad(x, t) \in D, \quad k=0,1, \quad k+m \leq 2 .
$$

Proof. See $[10,31]$.

Corollary 2. According to Theorem 3 upper bound for derivative of $y(x, t)$ relative to $x$ is infinite when $\varepsilon \rightarrow 0$ and $x \rightarrow 1$,

$$
\lim _{x \rightarrow 1, \varepsilon \rightarrow 0} \partial_{x} y(x, t) \rightarrow \infty .
$$

Corollary 3. According to Theorem 3 upper bound for derivative of $y(x, t)$ relative to $t$ is independent of the negative powers of the $\varepsilon$ and therefore it is finite.

We know that the solution of problem (1.1) and its derivative relative to $x$ $\left(\partial_{x} y(x, t)\right)$ has the boundary layer behavior. Moreover, in the boundary layer region, the derivative of the solution relative to $x$ is of great value, see Figure 7 
(Appendix). Therefore, in order to solve problem (1.1) region $D$ is split into two regions, so that one of them would contain a boundary layer behavior and uses a proper variable change in this region. It should be remarked that the present method is employed only to approach the solution of problem (1.1) and is not applicable to the approximate derivative of the solution, since on the boundary layer region, the value of the solution derivative $\left(\partial_{x} y(x, t)\right)$ is very high.

Remark 3. In summary, since the derivative of solution (1.1) relative to $x$ has the boundary layer behavior and its value is large, the present method is not appropriate to approach the derivation of the solution according to Corollary 2.

Remark 4. According to Remark 3, error analysis theorems are valid if, in the process of proving theorems, we do not use the derivative of the approximate solution $\left(\partial_{x} \ddot{y}_{\ddot{n}, n}(x, t)\right)$. Accordingly, in the present work the proof process for the error analysis theorems are provided without using the derivative of the approximate solution $\left(\partial_{x} \ddot{y}_{\ddot{n}, n}(x, t)\right)$, but using the $\partial_{t} \dot{y}_{\dot{n}, n}(x, t)$, instead. (Theorems 6,7$)$.

Remark 5. Since in the process of proving error analysis theorems, uniform convergence to the exact solution is required, the approximate solution is illustrated, and its derivation relative to $t\left(\partial_{t} y(x, t)\right)$ is uniformly convergent in Theorem 2 and Corollary 1.

\subsection{Error estimation}

Theorem 4. Suppose $\dot{y}_{\dot{n}, n}(x, t)$ is the approximate solution of the problem (2.5) in space $\mathcal{W}_{2}^{(3,2)}\left(D_{1}\right)$ and $y(x, t)$ is the exact solution, if $y(x, t) \in \mathcal{W}_{2}^{(3,2)}\left(D_{1}\right)$ then,

$$
\left\|y(x, t)-\dot{y}_{\dot{n}, n}(x, t)\right\|_{\infty} \leq C_{1} h_{x}+C_{2} h_{t},
$$

where $(x, t) \in D_{1}$ and $\left\|\dot{y}_{\dot{n}, n}(x, t)-y(x, t)\right\|_{\infty}=\max _{(x, t) \in D_{1}}\left|\dot{y}_{\dot{n}}(x, t)-y(x, t)\right|$ and $C_{1}, C_{2}$ are positive constants, $h_{x}=\max _{1 \leq i \leq \dot{n}_{1}}\left|x_{i+1}-x_{i}\right|$, and $h_{t}=$ $\max _{1 \leq j \leq \dot{n}_{2}}\left|t_{j+1}-t_{j}\right| . \quad \dot{n}=\dot{n}_{1} \times \dot{n}_{2}$ are number of collocation points in region $D_{1}$.

Proof. From $[7,8,13,19]$, and in each $\left[x_{i}, x_{i+1}\right] \times\left[t_{j}, t_{j+1}\right] \subset D_{1}$ we have,

$$
\begin{aligned}
& \left\|\mathcal{L}^{-1} R_{\dot{n}}(x, t)\right\|_{\infty}=\left\|y(x, t)-\dot{y}_{\dot{n}, n}(x, t)\right\|_{\infty} \\
& =\left\|y(x, t)-y\left(x_{i}, t_{j}\right)+\dot{y}_{\dot{n}, n}\left(x_{i}, t_{j}\right)-\dot{y}_{\dot{n}, n}(x, t)+y\left(x_{i}, t_{j}\right)-\dot{y}_{\dot{n}, n}\left(x_{i}, t_{j}\right)\right\|_{\infty} .
\end{aligned}
$$

By expansion of $y(x, t)$ at the point $\left(x_{i}, t_{j}\right)$ we have,

$$
\begin{aligned}
& y(x, t) \simeq y\left(x_{i}, t_{j}\right)+\left[\left(x-x_{i}\right) \partial_{x} y\left(x_{i}, t_{j}\right)+\left(t-t_{j}\right) \partial_{t} y\left(x_{i}, t_{j}\right)\right], \\
& \left|y(x, t)-y\left(x_{i}, t_{j}\right)\right| \leq\left|\left(x-x_{i}\right) \partial_{x} y\left(x_{i}, t_{j}\right)\right|+\left|\left(t-t_{j}\right) \partial_{t} y\left(x_{i}, t_{j}\right)\right|,
\end{aligned}
$$

since $y(x, t) \in \mathcal{W}_{2}^{(3,2)}\left(D_{1}\right)$, constants $\tilde{C}_{1}, \tilde{C}_{2}$ exist such that, $\forall(x, t) \in D_{1}$ $\left|\partial_{x} y(x, t)\right| \leq \tilde{C}_{1}$ and $\left|\partial_{t} y(x, t)\right| \leq \tilde{C}_{2}$ and we have,

$$
\left\|y(x, t)-y\left(x_{i}, t_{j}\right)\right\|_{\infty} \leq \tilde{C}_{1} h_{x}+\tilde{C}_{2} h_{t},
$$


moreover, we can write

$$
\begin{aligned}
& \dot{y}_{\dot{n}, n}\left(x_{i}, t_{j}\right)-\dot{y}_{\dot{n}, n}(x, t)=-\int_{x_{i}}^{x} \partial_{s} \dot{y}_{\dot{n}, n}\left(s, t_{j}\right) d s-\int_{t_{j}}^{t} \partial_{w} \dot{y}_{\dot{n}, n}(x, w) d w \\
& \left|\dot{y}_{\dot{n}, n}\left(x_{i}, t_{j}\right)-\dot{y}_{\dot{n}, n}(x, t)\right| \leq \int_{x_{i}}^{x}\left|\partial_{s} \dot{y}_{\dot{n}, n}\left(s, t_{j}\right)\right| d s+\int_{t_{j}}^{t}\left|\partial_{w} \dot{y}_{\dot{n}, n}(x, w)\right| d w,
\end{aligned}
$$

since $\dot{y}_{\dot{n}, n}(x, t) \in \mathcal{W}_{2}^{(3,2)}\left(D_{1}\right)$, constants $\hat{C}_{1}, \hat{C}_{2}$ exist such that, $\left|\partial_{x} \dot{y}_{\dot{n}, n}(x, t)\right| \leq$ $\hat{C}_{1}$ and $\left|\partial_{t} \dot{y}_{\dot{n}, n}(x, t)\right| \leq \hat{C}_{2}$ and therefore we have,

$$
\left\|\dot{y}_{\dot{n}, n}\left(x_{i}, t_{j}\right)-\dot{y}_{\dot{n}, n}(x, t)\right\|_{\infty} \leq \hat{C}_{1} h_{x}+\hat{C}_{2} h_{t} .
$$

From Theorem 2 we have $\dot{y}_{\dot{n}, n}(x, t) \longrightarrow y(x, t)$ when $\dot{n} \rightarrow \infty$, therefore for sufficiently large $\dot{n},\left|\dot{y}_{\dot{n}, n}\left(x_{i}, t_{j}\right)-y\left(x_{i}, t_{j}\right)\right|<\epsilon_{1}$ and by combining the above inequalities equations,

$$
\left\|R_{\dot{n}}(x, t)\right\|_{\infty} \leq \stackrel{\circ}{C}_{1} h_{x}+\stackrel{\circ}{C}_{2} h_{t}, \quad\left\|y(x, t)-\dot{y}_{\dot{n}, n}(x, t)\right\|_{\infty} \leq C_{1} h_{x}+C_{2} h_{t} .
$$

Theorem 5. Consider assumptions of the Theorem 4 then,

$$
\left\|\partial_{t} y(x, t)-\partial_{t} \dot{y}_{\dot{n}, n}(x, t)\right\|_{\infty} \leq K_{1} h_{x}+K_{2} h_{t},
$$

where $(x, t) \in D_{1}$ and $\left\|\partial_{t} \dot{y}_{\dot{n}, n}(x, t)-\partial_{t} y(x, t)\right\|_{\infty}=\max _{(x, t) \in D_{1}} \mid \partial_{t} \dot{y}_{\dot{n}, n}(x, t)-$ $\partial_{t} y(x, t) \mid$ and $K_{1}, K_{2}$ are positive constants.

Proof. Similar to Theorem 4 and in each $\left[x_{i}, x_{i+1}\right] \times\left[t_{j}, t_{j+1}\right] \subset D_{1}$ we have,

$$
\begin{aligned}
& \left\|\mathcal{L}^{-1} \partial_{t} R_{\dot{n}}(x, t)\right\|_{\infty}=\left\|\partial_{t} y(x, t)-\partial_{t} \dot{y}_{\dot{n}, n}(x, t)\right\|_{\infty}=\| \partial_{t} y(x, t)-\partial_{t} y\left(x_{i}, t_{j}\right) \\
& \quad+\partial_{t} \dot{y}_{\dot{n}, n}\left(x_{i}, t_{j}\right)-\partial_{t} \dot{y}_{\dot{n}, n}(x, t)+\partial_{t} y\left(x_{i}, t_{j}\right)-\partial_{t} \dot{y}_{\dot{n}, n}\left(x_{i}, t_{j}\right) \| .
\end{aligned}
$$

By expansion of $\partial_{t} y(x, t)$ at the point $\left(x_{i}, t_{j}\right)$ we have,

$$
\partial_{t} y(x, t)=\partial_{t} y\left(x_{i}, t_{j}\right)+\left[\left(x-x_{i}\right) \frac{\partial^{2} y\left(x_{i}, t_{j}\right)}{\partial x \partial t}+\left(t-t_{j}\right) \frac{\partial^{2} y\left(x_{i}, t_{j}\right)}{\partial t \partial x}\right],
$$

since $y(x, t) \in \mathcal{W}_{2}^{(3,2)}\left(D_{1}\right)$ there exists constant $\tilde{K}_{1}$ such that, $\left|\frac{\partial^{2} y(x, t)}{\partial x \partial t}\right| \leq \tilde{K}_{1}$,

$$
\left\|\partial_{t} y(x, t)-\partial_{t} y\left(x_{i}, t_{j}\right)\right\|_{\infty} \leq \tilde{K}_{1} h_{x}+\tilde{K}_{1} h_{t},
$$

moreover, we can write

$$
\partial_{t} \dot{y}_{\dot{n}, n}\left(x_{i}, t_{j}\right)-\partial_{t} \dot{y}_{\dot{n}, n}(x, t)=-\int_{x_{i}}^{x} \partial_{t} \partial_{s} \dot{y}_{\dot{n}, n}\left(s, t_{j}\right) d s-\int_{t_{j}}^{t} \partial_{w}^{2} \dot{y}_{\dot{n}, n}(x, w) d w,
$$

and since $\dot{y}_{\dot{n}, n}(x, t) \in \mathcal{W}_{2}^{(3,2)}\left(D_{1}\right)$ there exist constants $\hat{K}_{1}, \hat{K}_{2}$ where $\left|\partial_{t} \partial_{x} \dot{y}_{\dot{n}, n}(x, t)\right| \leq \hat{K}_{1},\left|\partial_{t}^{2} \dot{y}_{\dot{n}, n}(x, t)\right| \leq \hat{K}_{2}$ and therefore we have,

$$
\left\|\partial_{t} \dot{y}_{\dot{n}, n}\left(x_{i}, t_{j}\right)-\partial_{t} \dot{y}_{\dot{n}, n}(x, t)\right\|_{\infty} \leq \hat{K}_{1} h_{x}+\hat{K}_{2} h_{t} .
$$


From Theorem 2 and properties of reproducing kernel we have $\partial_{t} \dot{y}_{\dot{n}, n}(x, t) \longrightarrow$ $\partial_{t} y(x, t)$ when $\dot{n} \rightarrow \infty$, therefore for sufficiently large $\dot{n}, \mid \partial_{t} \dot{y}_{\dot{n}, n}\left(x_{i}, t_{j}\right)-$ $\partial_{t} y\left(x_{i}, t_{j}\right) \mid<\epsilon_{2}$ and by combining above equations,

$$
\left\|\partial_{t} R_{\dot{n}}(x, t)\right\|_{\infty} \leq \stackrel{\circ}{K}_{1} h_{x}+\stackrel{\circ}{K}_{2} h_{t}, \quad\left\|\partial_{t} y(x, t)-\partial_{t} \dot{y}_{\dot{n}, n}(x, t)\right\|_{\infty} \leq K_{1} h_{x}+K_{2} h_{t} .
$$

Now, in order to obtain error estimation between $\ddot{y}_{\ddot{n}, n}(x, t)$ and $y(x, t)$ on the region $D_{2}$, first provide some essential preliminaries, so consider equation (2.6) as follows [15],

$$
\left\{\begin{array}{l}
-\varepsilon \partial_{x}^{2} y(x, t)+p(x, t) \partial_{x} y(x, t)+q(x, t) y(x, t)+\partial_{t} y(x, t) \\
=\mathcal{N}(y(x, t))+f(x, t), \quad(x, t) \in D_{2}, \\
y(1, t)=0, \quad y(1-\mu, t)=\dot{y}_{\dot{n}, n}(1-\mu, t), \quad t \in[0,1] \\
y(x, 0)=y_{0}(x), \quad x \in[1-\mu, 1] .
\end{array}\right.
$$

Consider following assumptions and change region $D_{2}$ into $D_{3}, x=\mu z+1$ and $y(x, t)=u(z, t)$ such that $\partial_{z} u(z, t)=\mu \partial_{x} y(x, t)$ and $\partial_{z}^{2} u(z, t)=\mu^{2} \partial_{x}^{2} y(x, t)$ therefore the equation (3.7) turns into,

$$
\left\{\begin{array}{l}
\frac{-\varepsilon}{\mu^{2}} \partial_{z}^{2} u(z, t)+\frac{1}{\mu} p(\mu z+1, t) \partial_{z} u(z, t)+q(\mu z+1, t) u(z, t)+\partial_{t} u(z, t) \\
=\mathcal{N}(u(z, t))+f(\mu z+1, t), \quad(z, t) \in D_{3} \equiv[-1,0] \times[0,1] \\
u(0, t)=0, \quad u(-1, t)=\dot{y}_{\dot{n}, n}(1-\mu, t), \quad t \in[0,1] \\
u(z, 0)=u_{0}(z), \quad z \in[-1,0]
\end{array}\right.
$$

Now by homogenizing the initial and boundary conditions of problem (3.8) with function $u(z, t)=\bar{u}(z, t)+H_{1}(z, t)$ such that $H_{1}(z, 0)=u_{0}(z)=y_{0}(\mu z+1)$ and $H_{1}(-1, t)=\dot{y}_{\dot{n}, n}(1-\mu, t)$, therefore we have following equation,

$$
\left\{\begin{array}{l}
\quad \frac{-\varepsilon}{\mu^{2}} \partial_{z}^{2} \bar{u}(z, t)+\frac{1}{\mu} p(\mu z+1, t) \partial_{z} \bar{u}(z, t)+q(\mu z+1, t) \bar{u}(z, t)+\partial_{t} \bar{u}(z, t) \\
=\mathcal{N}(\bar{u}(z, t))+\bar{f}(\mu z+1, t), \quad(z, t) \in D_{3} \equiv[-1,0] \times[0,1] \\
\bar{u}(0, t)=0, \quad \bar{u}(-1, t)=0, \quad t \in[0,1] \\
\bar{u}(z, 0)=0, \quad z \in[-1,0] .
\end{array}\right.
$$

Suppose $\underline{u}(z, t)$ is the approximate solution of the problem (3.9) that has been obtained using RKM in space $\mathcal{W}_{2}^{(3,2)}\left(D_{3}\right)$, therefore

$$
\ddot{y}_{\ddot{n}, n}(x, t)=\underline{u}((x-1) / \mu, t)+H_{1}((x-1) / \mu, t) .
$$

Consider the following equation,

$$
\left\{\begin{array}{l}
-\varepsilon \partial_{x}^{2} v(x, t)+p(x, t) \partial_{x} v(x, t)+q(x, t) v(x, t)+\partial_{t} v(x, t) \\
=\mathcal{N}(v(x, t))+g(x, t), \quad(x, t) \in D_{2}, \\
v(1, t)=0, \quad v(1-\mu, t)=y(1-\mu, t), \quad t \in[0,1] \\
v(x, 0)=v_{0}(x), \quad x \in[1-\mu, 1]
\end{array}\right.
$$

It is clear that the solution of the problem $(3.10)$ is $y(x, t)$ where $(x, t) \in D_{2}$. By using the following variable change, $x=\mu z+1$ and $v(x, t)=\nu(z, t)$ such 
that $\partial_{z} \nu(z, t)=\mu \partial_{x} v(x, t)$ and $\partial_{z}^{2} \nu(z, t)=\mu^{2} \partial_{x}^{2} v(x, t)$, we have shifted problem (3.8) to the problem (3.10),

$$
\left\{\begin{array}{l}
\frac{-\varepsilon}{\mu^{2}} \partial_{z}^{2} \nu(z, t)+\frac{1}{\mu} p(\mu z+1, t) \partial_{z} \nu(z, t)+q(\mu z+1, t) \nu(z, t)+\partial_{t} \nu(z, t) \\
=\mathcal{N}(\nu(z, t))+g(\mu z+1, t), \quad(z, t) \in D_{3} \equiv[-1,0] \times[0,1] \\
\nu(0, t)=0, \quad \nu(-1, t)=y(1-\mu, t), \quad t \in[0,1] \\
\nu(z, 0)=\nu_{0}(z), \quad z \in[-1,0] .
\end{array}\right.
$$

Therefore we homogenize obtained problem (3.11), with $\nu(z, t)=\bar{\nu}(z, t)+$ $H_{2}(z, t)$ such that $H_{2}(z, 0)=\nu_{0}(z)=v_{0}(\mu z+1)$ and $H_{2}(-1, t)=y(1-\mu, t)$, therefore problem (3.11) turns to,

$$
\left\{\begin{array}{l}
\frac{-\varepsilon}{\mu^{2}} \partial_{z}^{2} \bar{\nu}(z, t)+\frac{1}{\mu} p(\mu z+1, t) \partial_{z} \bar{\nu}(z, t)+q(\mu z+1, t) \bar{\nu}(z, t)+\partial_{t} \bar{\nu}(z, t) \\
=\mathcal{N}(\bar{\nu}(z, t))+\bar{g}(\mu z+1, t), \quad(z, t) \in D_{3} \equiv[-1,0] \times[0,1] \\
\bar{\nu}(0, t)=0, \quad \bar{\nu}(-1, t)=0, \quad t \in[0,1] \\
\bar{\nu}(z, 0)=0, \quad z \in[-1,0]
\end{array}\right.
$$

and suppose $\underline{\nu}(z, t)$ is the approximate solution of the problem (3.12) that has been obtained using RKM in space $\mathcal{W}_{2}^{(3,2)}\left(D_{3}\right)$.

Theorem 6. Suppose $\underline{u}(z, t)$ is the approximate solution of the equation (3.9) and $\bar{\nu}(z, t)$ is the solution of the equation (3.12) and $p(\mu z+1, t), q(\mu z+1, t) \in$ $\mathcal{C}^{2}\left(D_{3}\right)$ then,

$$
\|\underline{u}(z, t)-\bar{\nu}(z, t)\|_{\infty} \leq M_{1} h_{x}+M_{2} h_{t}
$$

where $\left(x_{i}, t_{j}\right) \in D_{3}$ and $\|\underline{u}(z, t)-\bar{\nu}(z, t)\|_{\infty}=\max _{(x, t) \in D_{3}}=|\underline{u}(z, t)-\bar{\nu}(z, t)|$ and $M_{1}, M_{2}$ are constants, $h_{x}=\max _{1 \leq i \leq \ddot{n}_{1}}\left|x_{i+1}-x_{i}\right|$, and $h_{t}=\max _{1 \leq j \leq \ddot{n}_{2}}$ $\left|t_{j+1}-t_{j}\right|$. Number of collocation points in region $D_{3}$ are $\ddot{n}=\ddot{n}_{1} \times \ddot{n}_{2}$.

Proof. Since $\underline{u}(z, t), \bar{\nu}(z, t)$ are approximate solutions of the equations (3.9) and (3.12), respectively, therefore we have

$$
\begin{aligned}
& \left\|R_{\ddot{n}}(z, t)\right\|_{\infty}=\|\mathcal{L} \underline{u}(z, t)-\mathcal{L} \bar{\nu}(z, t)\|_{\infty}=\| \mathcal{L} \underline{u}(z, t)-\bar{f}(\mu z+1, t)+\bar{f}(\mu z+1, t) \\
& -\mathcal{L} \bar{\nu}(z, t)\left\|_{\infty} \leq\right\| \mathcal{L} \underline{u}(z, t)-\bar{f}(\mu z+1, t)\left\|_{\infty}+\right\| \bar{f}(\mu z+1, t)-\bar{g}(\mu z+1, t) \|_{\infty} .
\end{aligned}
$$

By using Theorems 4 it is clear that $\|\mathcal{L} \underline{u}(z, t)-\bar{f}(\mu z+1, t)\|_{\infty} \max _{(x, t) \in D_{3}}=$ $|\mathcal{L} \underline{u}(z, t)-\bar{f}(\mu z+1, t)| \leq \hat{M}_{1} h_{x}+\hat{M}_{2} h_{t}$. From problem $(3.8)$ and $u(z, t)=$ $\underline{u}(z, t)+H_{1}(z, t)$ we have,

$$
\begin{aligned}
\bar{f}(\mu z+1, t)= & \frac{-\varepsilon}{\mu^{2}} \partial_{z}^{2} H_{1}(z, t)+\frac{1}{\mu} p(\mu z+1, t) \partial_{z} H_{1}(z, t)+q(\mu z+1, t) H_{1}(z, t) \\
& +\partial_{t} H_{1}(z, t) .
\end{aligned}
$$

Using the problem $(3.11)$ and $\nu(z, t)=\bar{\nu}(z, t)+H_{2}(z, t)$ we have,

$$
\begin{aligned}
\bar{g}(\mu z+1, t)= & \frac{-\varepsilon}{\mu^{2}} \partial_{z}^{2} H_{2}(z, t)+\frac{1}{\mu} p(\mu z+1, t) \partial_{z} H_{2}(z, t)+q(\mu z+1, t) H_{2}(z, t) \\
& +\partial_{t} H_{2}(z, t)
\end{aligned}
$$


it is easy to see that homogenization functions $H_{1}(z, t), H_{2}(z, t)$ can be defined in the from, $H_{1}(z, t)=a(z, t) u_{0}(z)+b(z, t) \dot{y}_{\dot{n}, n}(1-\mu, t)$ and $H_{2}(z, t)=$ $a(z, t) \nu_{0}(z)+b(z, t) y(1-\mu, t)$ such that $a(z, 0)=1, b(z, 0)=0$ and $a(-1, t)=$ $0, b(-1, t)=1$ therefore we have,

$$
\begin{aligned}
& \partial_{z}^{2} H_{1}(z, t)=a_{1}(z, t)+\dot{y}_{\dot{n}, n}(1-\mu, t) \partial_{z}^{2} b(z, t), \\
& \partial_{z} H_{1}(z, t)=a_{2}(z, t)+\dot{y}_{\dot{n}, n}(1-\mu, t) \partial_{z} b(z, t), \\
& \partial_{t} H_{1}(z, t)=a_{3}(z, t)+\dot{y}_{\dot{n}, n}(1-\mu, t) \partial_{t} b(z, t)+b(z, t) \partial_{t} \dot{y}_{\dot{n}, n}(1-\mu, t), \\
& \partial_{z}^{2} H_{2}(z, t)=a_{1}(z, t)+y(1-\mu, t) \partial_{z}^{2} b(z, t), \\
& \partial_{z} H_{2}(z, t)=a_{2}(z, t)+y(1-\mu, t) \partial_{z} b(z, t), \\
& \partial_{t} H_{2}(z, t)=a_{3}(z, t)+y(1-\mu, t) \partial_{t} b(z, t)+b(z, t) \partial_{t} y(1-\mu, t),
\end{aligned}
$$

also $|p(\mu z+1, t)| \leq m_{1},|q(\mu z+1, t)| \leq m_{2}$ and we can write,

$$
\begin{gathered}
\|\bar{f}(\mu z+1, t)-\bar{g}(\mu z+1, t)\|_{\infty} \leq \frac{-\varepsilon}{\mu^{2}}\left\|\partial_{z}^{2} H_{1}(z, t)-\partial_{z}^{2} H_{2}(z, t)\right\|_{\infty}+\frac{m_{1}}{\mu} \| \partial_{z} H_{1}(z, t) \\
-\partial_{z} H_{2}(z, t)\left\|_{\infty}+m_{2}\right\| H_{1}(z, t)-H_{2}(z, t)\left\|_{\infty}+\right\| \partial_{t} H_{1}(z, t)-\partial_{t} H_{2}(z, t) \|_{\infty},
\end{gathered}
$$

where,

$$
\begin{aligned}
& \left\|H_{1}(z, t)-H_{2}(z, t)\right\|_{\infty}=\| a(z, t) u_{0}(z)-a(z, t) \nu_{0}(z)+b(z, t) \dot{y}_{\dot{n}, n}(1-\mu, t) \\
& \quad-b(z, t) y(1-\mu, t)\left\|_{\infty} \leq\right\| a(z, t) u_{0}(z)-a(z, t) \nu_{0}(z) \|_{\infty} \\
& \quad+\left\|b(z, t) \dot{y}_{\dot{n}, n}(1-\mu, t)-b(z, t) y(1-\mu, t)\right\|_{\infty} \leq \tilde{M}_{1} h_{x}+\tilde{M}_{2} h_{t} .
\end{aligned}
$$

Similar to equation (3.14) and using Theorems 4,5 we have, $\| \bar{f}(\mu z+1, t)-$ $\bar{g}(\mu z+1, t) \|_{\infty} \leq \check{M}_{1} h_{x}+\check{M}_{2} h_{t}$, and therefore we have

$$
\left\|R_{\ddot{n}}(z, t)\right\|_{\infty}=\|\mathcal{L} \underline{u}(z, t)-\mathcal{L} \bar{\nu}(z, t)\|_{\infty} \leq \stackrel{\circ}{M}_{1} h_{x}+\stackrel{\circ}{M}_{2} h_{t}
$$

and from Theorem 4 it is clear $\|\underline{u}(z, t)-\bar{\nu}(z, t)\|_{\infty} \leq M_{1} h_{x}+M_{2} h_{t}$.

Theorem 7. Suppose $\ddot{y}_{\ddot{n}, n}(x, t)$ is the approximate solution of the problem (2.6) in space $\mathcal{W}_{2}^{(3,2)}\left(D_{3}\right)$ and $y(x, t)$ is the exact solution, if $y(x, t) \in \mathcal{W}_{2}^{(3,2)}(D)$ then,

$$
\left\|y(x, t)-\ddot{y}_{\ddot{n}, n}(x, t)\right\|_{\infty} \leq C_{3} h_{x}+C_{4} h_{t},
$$

where $\left(x_{i}, t_{j}\right) \in D_{3}$ and $\left\|y(x, t)-\ddot{y}_{\ddot{n}, n}(x, t)\right\|_{\infty}=\max _{(x, t) \in D_{3}}=\mid y(x, t)-$ $\ddot{y}_{\ddot{n}, n}(x, t) \mid$ and $C_{3}, C_{4}$ are constants, $h_{x}=\max _{1 \leq i \leq \ddot{n}_{1}}\left|x_{i+1}-x_{i}\right|$, and $h_{t}=$ $\max _{1 \leq j \leq \ddot{n}_{2}}\left|t_{j+1}-t_{j}\right|$. Number of collocation points in region $D_{3}$ are $\ddot{n}=$ $\ddot{n}_{1} \times \ddot{n}_{2}$.

Proof. From mentioned preliminaries, $\underline{u}(z, t)$ is approximate solution of equation (3.9) and $y(x, t)$ is exact solution of equation (3.10) and $\ddot{y}_{\ddot{n}, n}(x, t)=$ $\underline{u}(z, t)+H_{1}(z, t), y(x, t)=\bar{\nu}(z, t)+H_{2}(z, t)$ where $z=\frac{x-1}{\mu}$ therefore we have,

$$
\begin{aligned}
\left\|y(x, t)-\ddot{y}_{\ddot{n}, n}(x, t)\right\|_{\infty} & =\left\|\underline{u}(z, t)+H_{1}(z, t)-\bar{\nu}(z, t)-H_{2}(z, t)\right\|_{\infty} \\
& \leq\|\underline{u}(z, t)-\bar{\nu}(z, t)\|_{\infty}+\left\|H_{1}(z, t)-H_{2}(z, t)\right\|_{\infty},
\end{aligned}
$$

using Theorem 6 the proof is completed. 
Theorem 8. Suppose $\mathcal{Y}_{N, n}(x, t)$ is the approximate solution of the problem (1.1) that has been obtained using RKM in space $\mathcal{W}_{2}^{(3,2)}$ and $y(x, t)$ is the exact solution, if $p(x, t), q(x, t) \in \mathcal{C}^{2}(D)$ and $D \equiv[0,1] \times[0,1]$ then,

$$
\left\|\mathcal{Y}_{N, n}(x, t)-y(x, t)\right\|_{\infty}=\max _{(x, t) \in D}\left|\mathcal{Y}_{N, n}(x, t)-y(x, t)\right| \leq C_{x} h_{x}+C_{t} h_{t},
$$

where $\left(x_{i}, t_{j}\right) \in D$ and $C_{x}, C_{t}$ are constants, $h_{x}=\max _{1 \leq i \leq N}\left|x_{i+1}-x_{i}\right|$, and $h_{t}=\max _{1 \leq j \leq N}\left|t_{j+1}-t_{j}\right|$. Number of collocation points in throughout region $D$ is $N$, and $N=\dot{n}+\ddot{n}$.

Proof. By using Theorems 4 and 7 and definition of $\mathcal{Y}_{N, n}(x, t)$ on the region $D$,

$$
\mathcal{Y}_{N, n}(x, t)= \begin{cases}\dot{y}_{\dot{n}, n}(x, t) & (x, t) \in D_{1}, \\ \ddot{y}_{\ddot{n}, n}(x, t) & (x, t) \in D_{2},\end{cases}
$$

and the proof is completed.

\section{Numerical results}

We consider the following numerical examples [17],

Example 1. $-\varepsilon \partial_{x}^{2} y(x, t)+\left(1+x-x^{2}\right) \partial_{x} y(x, t)+\partial_{t} y(x, t)=f_{1}(x, t)$.

Example 2. $-\varepsilon \partial_{x}^{2} y(x, t)+\left(-x^{2}+x+1\right) \partial_{x} y(x, t)+\partial_{t} y(x, t)+e^{y(x, t)}=f_{2}(x, t)$.

Example 3. $-\varepsilon \partial_{x}^{2} y(x, t)+\left(-x^{2}+x+1\right) \partial_{x} y(x, t) y(x, t)+\partial_{t} y(x, t)+e^{y(x, t)}=$ $f_{3}(x, t)$.

Example 4. $-\varepsilon \partial_{x}^{2} y(x, t)+\left(2-x^{2}\right) \partial_{x} y(x, t)+x y(x, t)+\partial_{t} y(x, t)=10 t^{2} e^{-t} x(1-x)$.

For Examples 1, 2 and 3, the exact solution is, $y(x, t)=e^{-t}\left(x\left(1-e^{-1 / \epsilon}\right)\right.$ $\left.-e^{\frac{x-1}{\epsilon}}+e^{-1 / \epsilon}\right)$ where $y_{0}(x)=x\left(1-e^{-1 / \epsilon}\right)-e^{\frac{x-1}{\epsilon}}+e^{-1 / \epsilon}$ and for Example 4 the exact solution is not known and $y_{0}(x)=0$. See Figure 1 for Example 1, Figure 2 for Example 2, Figure 3 for Example 3, and Figure 4 for Example 4 which they show the maximum absolute error for various $\epsilon$. If perturbation parameter is not too small $\left(\varepsilon=1,10^{-2}\right)$, then nonuniform collocation points are $\left(1-\left(\frac{i}{n_{1}+\frac{1}{3}}\right)^{2}, \frac{j}{n_{2}+\frac{1}{2}}\right)$, in throughout region $D$ where $i=1,2, \ldots, n_{1}, j=1,2, \ldots, n_{2}$. Otherwise $\left(\varepsilon=10^{-4,-6,-8, \ldots}\right)$ nonuniform collocation points for boundary layer region, are $\left(-\left(\frac{i}{\ddot{n}_{1}+\frac{1}{3}}\right)^{2}, \frac{j}{\ddot{n}_{2}+\frac{1}{2}}\right)$ throughout $D_{3}$ where $i=1,2, \ldots, \ddot{n}_{1}, j=1,2, \ldots, \ddot{n}_{2}$, and collocation points for the regular region are $\left(1-\left(\frac{i}{\dot{n}_{1}+\frac{1}{7}}\right)^{2},\left(\frac{j}{\dot{n}_{2}+\frac{1}{6}}\right)^{2}\right)$ throughout $D_{1}$, where $i=1,2, \ldots, \dot{n}_{1}, j=$ $1,2, \ldots, \dot{n}_{2}$. Examples 1,2 and 3 have the same exact solution, see Figure 5 for different values of $\epsilon$, and Figure 6 for Example 4. All present numerical results are obtained using Wolfram Mathematica 10 software.

Remark 6. For solving problem (1.1), first we divided $D$ to regular region $D_{1} \equiv[0,1-\mu] \times[0,1]$ and boundary layer region $D_{2} \equiv[1-\mu, 1] \times[0,1]$ 
and we shifted region $D_{2}$ into $D_{3} \equiv[-1,0] \times[0,1]$ and obtain the reproducing kernels $\dot{\mathcal{K}}_{\eta, \xi}(x, t)$ and $\ddot{\mathcal{K}}_{\eta, \xi}(x, t)$ for regular and shifted boundary layer regions, respectively. Then we construct complete functional systems $\varphi_{i j}(x, t)=$ $\left.\dot{\mathcal{K}}_{\eta, \xi}(x, t)\right|_{(\eta, \xi)=\left(x_{i}, t_{j}\right)}$ and $\varphi_{i j}(x, t)=\left.\ddot{\mathcal{K}}_{\eta, \xi}(x, t)\right|_{(\eta, \xi)=\left(x_{i}, t_{j}\right)}$ for reproducing kernel spaces $\mathcal{W}_{2}^{(3,2)}\left(D_{1}\right)$ and $\mathcal{W}_{2}^{(3,2)}\left(D_{3}\right)$ respectively. For regular region $D_{1}$, $\dot{n}=\dot{n}_{1} \times \dot{n}_{2}$ where $i=1,2, \ldots, \dot{n}_{1}$ and $j=1,2, \ldots, \dot{n}_{2}$. For shifted boundary layer region $D_{3}, \ddot{n}=\ddot{n}_{1} \times \ddot{n}_{2}$ where $i=1,2, \ldots, \ddot{n}_{1}$ and $j=1,2, \ldots, \ddot{n}_{2}$ and $\ell \equiv i, j$. Therefor we solve problem (1.1) in the regular and shifted boundary layer region such that approximate solutions are as following form,

$$
\dot{y}_{\dot{n}, n}(x, t)=\sum_{\ell=1}^{\dot{n}} \varrho_{\ell, \dot{n}, n} \varphi_{\ell}(x, t), \quad \ddot{y}_{\ddot{n}, n}(x, t)=\sum_{\ell=1}^{\ddot{n}} \varrho_{\ell, \ddot{n}, n} \varphi_{\ell}(x, t),
$$

where we obtain coefficients $\varrho_{\ell, \dot{n}, n}$ and $\varrho_{\ell, \ddot{n}, n}$ by solving following system of algebraic equations

$$
\begin{aligned}
& \left.\sum_{\ell=1}^{\dot{n}} \varrho_{\ell, \dot{n}, n} \mathcal{L} \varphi_{\ell}(x, t)\right|_{(x, t)=\left(x_{i}, t_{j}\right)}=\left.\mathcal{N}\left(\dot{y}_{\dot{n}, n-1}(x, t)\right)\right|_{(x, t)=\left(x_{i}, t_{j}\right)}+f\left(x_{i}, t_{j}\right), \\
& \left.\sum_{\ell=1}^{\ddot{n}} \varrho_{\ell, \ddot{n}, n} \mathcal{L} \varphi_{\ell}(x, t)\right|_{(x, t)=\left(x_{i}, t_{j}\right)}=\left.\mathcal{N}\left(\ddot{y}_{\ddot{n}, n-1}(x, t)\right)\right|_{(x, t)=\left(x_{i}, t_{j}\right)}+f\left(x_{i}, t_{j}\right),
\end{aligned}
$$

where $n=2,3, \ldots$ is number of iterative for nonlinear term $\mathcal{N}\left(\dot{y}_{\dot{n}, n-1}(x, t)\right)$ and $\mathcal{N}\left(\ddot{y}_{\ddot{n}, n-1}(x, t)\right)$ with initial functions $\dot{y}_{\dot{n}, 0}(x, t)$ and $\ddot{y}_{\ddot{n}, 0}(x, t)$ for $n=1$ respectively.

Remark 7. According to Corollary 3 and Remark 3 we compared maximum absolute errors for derivative of the approximate solutions relative to $t$ $\left(\mathbb{E}^{\partial_{t} \mathcal{Y}_{N, n}(x, t)}=\operatorname{Max}\left|\partial_{t} \mathcal{Y}_{N, n}(x, t)-\partial_{t} y(x, t)\right|\right)$ and $x$ $\left(\mathbb{E}^{\partial_{x} \mathcal{Y}_{N, n}(x, t)}=\operatorname{Max}\left|\partial_{x} \mathcal{Y}_{N, n}(x, t)-\partial_{x} y(x, t)\right|\right)$ throughout $D$. We presented results in Table 3.

Remark 8. According to error analysis Theorems errors ratio for approximate solution $\mathcal{Y}_{N, n}(x, t)$ throughout $D$ must be about 0.5 . We showed errors ratio in Tables 1 and 2 for all numerical examples.

\section{Conclusions}

Hereby, a technique is introduced based on RKM to solve SPCDPPs. One of the advantages of this technique is that it could be used to solve SPCDPPs, so that SPCDPPs would not be easily solved employing common numerical methods or numerical commands in mathematical softwares that are available for free. Numerical examples demonstrated that the present method has higher precision compared to other methods. That is in account of the fact that the Gram-Schmidt process is removed. 


\section{Acknowledgements}

The authors are very grateful to anonymous reviewer for carefully reading the paper and for very useful discussions and suggestions which have improved the paper.

\section{References}

[1] A. Akgül. A novel method for a fractional derivative with nonlocal and non-singular kernel. Chaos. Solit. Fract., 114:478-482, 2018. https://doi.org/10.1016/j.chaos.2018.07.032.

[2] A. Akgül. Reproducing kernel Hilbert space method based on reproducing kernel functions for investigating boundary layer flow of a PowellEyring non-Newtonian fluid. J. of Taibah. Uni. for Sci., 13:858-863, 2019. https://doi.org/10.1080/16583655.2019.1651988.

[3] A. Akgül. Solutions of the linear and nonlinear differential equations within the generalized fractional derivatives. Chaos. Interdiscipl. J. Nonlinear. Sci., 29(2):023108, 2019. https://doi.org/0.1063/1.5084035.

[4] A. Akgül, A. Cordero and J.R. Torregrosa. Solutions of fractional gas dynamics equation by a new technique. Math. Meth. in the Appl. Sci, 43:1349-1358, 2020. https://doi.org/10.1002/mma.5950.

[5] A. Akgül and M. Modanli. Crank-Nicholson difference method and reproducing kernel function for third order fractional differential equations in the sense of Atangana-Baleanu Caputo derivative. Chaos. Solit. Fract., 127:10-16, 2019. https://doi.org/10.1016/j.chaos.2019.06.011.

[6] B. Azarnavid, F. Parvaneh and S. Abbasbandy. Picard-reproducing kernel Hilbert space method for solving generalized singular nonlinear Lane-Emden type equations. Math. Model. Anal., 20:754-767, 2015. https://doi.org/10.3846/13926292.2015.1111953.

[7] E. Babolian and D. Hamedzadeh. A splitting iterative method for solving second kind integral equations in reproducing kernel spaces. J. Comput. Appl. Math., 326:204-216, 2017. https://doi.org/10.1016/j.cam.2017.05.025.

[8] E. Babolian, S. Javadi and E. Moradi. Error analysis of reproducing kernel Hilbert space method for solving functional integral equations. J. Comput. Appl. Math., 300:300-311, 2016. https://doi.org/10.1016/j.cam.2016.01.008.

[9] D. Baleanu, A. Fernandez and A. Akgül. On a fractional operator combining proportional and classical differintegrals. Mathematics, 8(3):360, 2020. https://doi.org/10.3390/math8030360.

[10] C. Clavero, J.C. Jorge and F. Lisbona. A uniformly convergent scheme on a nonuniform mesh for convection-diffusion parabolic problems. J. Comput. Appl. Math., 154:415-429, 2003. https://doi.org/10.1016/S0377-0427(02)00861-0.

[11] M.G. Cui and Y. Lin. Nonlinear Numerical Analysis in the Reproducing Kernel Space. Nova Science-Hauppauge-New York-United States, 2009.

[12] R.E. Ewing and H. Wang. A summary of numerical methods for time dependent advection-dominated partial differential equations. J. Comp. Appl. Math., 128:423-445, 2001. https://doi.org/10.1016/B978-0-444-50616-0.50018-X. 
[13] M. Fardi and M. Ghasemi. Solving nonlocal initial-boundary value problems for parabolic and hyperbolic integro-differential equations in reproducing kernel Hilbert space. Wiley. Online. Library., 33:174-198, 2017. https://doi.org/10.1002/num.22079.

[14] F.Z. Geng and S.P. Qian. Reproducing kernel method for singularly perturbed turning point problems having twin boundary layers. Appl. Math. Lett., 26:9981004, 2013. https://doi.org/10.1016/j.aml.2013.05.006.

[15] F.Z. Geng and S.P. Qian. Piecewise reproducing kernel method for singularly perturbed delay initial value problems. Appl. Math. Lett., 37:67-71, 2014. https://doi.org/10.1016/j.aml.2014.05.014.

[16] F.Z. Geng and S.P. Qian. Modified reproducing kernel method for singularly perturbed boundary value problems with a delay. Appl. Math. Model., 39:55925597, 2015. https://doi.org/10.1016/j.apm.2015.01.021.

[17] S. Gowrisankar and S. Natesan. Robust numerical scheme for singularly perturbed convection-diffusion parabolic initial-boundary-value problems on equidistributed grids. Comput. Phys. Commun., 185:2008-2019, 2014. https://doi.org/10.1016/j.cpc.2014.04.004.

[18] W. Hundsdorfer and J. Verwer. Numerical solution of time-dependent advectiondiffusion-reaction equations, volume 33 of Springer Series in Computational Mathematics. Springer-Verlag, Berlin, 2003. https://doi.org/10.1007/978-3-66209017-6.

[19] R. Ketabchi, R.Mokhtari and E.Babolian. Some error estimates for solving Volterra integral equations by using the reproducing kernel method. J. Comput. Appl. Math., 273:245-250, 2015. https://doi.org/10.1016/j.cam.2014.06.016.

[20] H.O. Kreiss and J. Lorenz. Initial-boundary value problems and the Navier-Stokes equations. Classics in Appl. Math. SIAM., 47, 2004. https://doi.org/10.1137/1.9780898719130.

[21] Z.Y. Li, Y.L. Wang, F.G. Tan, X.H. Wan, H. Yu and J.S. Duan. Solving a class of linear nonlocal boundary value problems using the reproducing kernel. Appl. Math. Comput., 265:1098-1105, 2015. https://doi.org/10.1016/j.amc.2015.05.117.

[22] T. Linß. Layer-Adapted Meshes for Reaction-Convection-Diffusion Problems. Lecture Notes in Mathematics-Springer-Verlag-Berlin-Heidelberg, 1985.

[23] P.A. Markowich, C. Ringhofer and S. Schmeiser. Semiconductor Equations. Springer-Vienna, 1990. https://doi.org/10.1007/978-3-7091-6961-2.

[24] H.G. Roos, M. Stynes and L. Tobiska. Robust Numerical Methods for Singularly Perturbed Differential Equations, Convection-Diffusion-Reaction and Flow Problems. Springer-Verlag Berlin Heidelberg, 2008.

[25] H. Sahihi, T. Allahviranloo and S. Abbasbandy. Solving system of second-order BVPs using a new algorithm based on reproducing kernel Hilbert space. Appl. Num. Math., 151:27-39, 2020. https://doi.org/10.1016/j.apnum.2019.12.008.

[26] M.G. Sakar. Iterative reproducing kernel Hilbert spaces method for Riccati differential equations. J. Comput. Appl. Math., 309:163-174, 2017. https://doi.org/10.1016/j.cam.2016.06.029.

[27] M.G. Sakar, O. Saldr and F. Erdogan. An iterative approximation for timefractional Cahn-Allen equation with reproducing kernel method. Comput. Appl. Math., 37:5951-5964, 2018. https://doi.org/10.1007/s40314-018-0672-9. 
[28] M.G. Sakar, O. Saldr and F. Erdogan. A hybrid method for singularly perturbed convection-diffusion equation. Int. J. Appl. Comput. Math., 5(135), 2019. https://doi.org/10.1007/s40819-019-0714-z.

[29] O. Saldr, M.G. Sakar and F. Erdogan. Numerical solution of time-fractional Kawahara equation using reproducing kernel method with error estimate. Comput. Appl. Math., 38(198), 2019. https://doi.org/10.1007/s40314-019-0979-1.

[30] R. Salmon. Lectures on Geophysical Fluid Dynamics. Oxford University PressNew York, 1998. https://doi.org/10.1093/oso/9780195108088.001.0001.

[31] M. Stynes and E. ORiordan. Uniformly convergent difference schemes for singularly perturbed parabolic diffusion-convection problems without turning points. Numer. Math., 55:521-544, 1989. https://doi.org/10.1007/BF01398914.

[32] Y. Wang, T. Chaolu and Z. Chen. Using reproducing kernel for solving a class of singular weakly nonlinear boundary value problems. Int. J. Comput. Mathe., 87:367-380, 2010. https://doi.org/10.1080/00207160802047640.

[33] Y. Wang, T. Chaolu and P. Jing. New algorithm for second-order boundary value problems of integro-differential equation. J. Comp. Appl. Math., 229:1-6, 2009. https://doi.org/10.1016/j.cam.2008.10.007.

[34] Y. Wang, L. Su, X. Cao and X. Li. Using reproducing kernel for solving a class of singularly perturbed problems. Comput. Math. Appl., 61:421-430, 2011. https://doi.org/10.1016/j.camwa.2010.11.019.

\section{Appendix: tables and figures}

Table 1. Max point-wise error $\left(\mathbb{E}^{\mathcal{Y}_{N, n}(x, t)}=\operatorname{Max}\left|\mathcal{Y}_{2 N, n}(x, t)-\mathcal{Y}_{N, n}(x, t)\right|,(x, t) \in D\right)$ and error ratio $\mathbb{E}^{\mathcal{Y}_{2 N, n}} / \mathbb{E}^{\mathcal{Y}_{N, n}}$ for Example 4 .

\begin{tabular}{|c|c|c|c|c|c|}
\hline \multirow[b]{2}{*}{$N$} & \multicolumn{3}{|l|}{ PM } & \multicolumn{2}{|l|}{$[17]$} \\
\hline & 200 & 400 & 800 & $512 \times 160$ & $1024 \times 320$ \\
\hline$\varepsilon$ & & & & & \\
\hline $10^{0}$ & $\begin{array}{c}7.00 \times 10^{-5} \\
0.257143\end{array}$ & $\begin{array}{c}1.80 \times 10^{-5} \\
0.472222\end{array}$ & $8.50 \times 10^{-6}$ & $6.21 \times 10^{-5}$ & $3.13 \times 10^{-5}$ \\
\hline $10^{-2}$ & $\begin{array}{c}2.80 \times 10^{-3} \\
0.250\end{array}$ & $\begin{array}{c}7.00 \times 10^{-4} \\
0.428571\end{array}$ & $3.00 \times 10^{-4}$ & $6.07 \times 10^{-4}$ & $3.10 \times 10^{-4}$ \\
\hline $10^{-4}$ & $\begin{array}{c}3.20 \times 10^{-3} \\
0.50\end{array}$ & $\begin{array}{c}1.60 \times 10^{-3} \\
0.2125\end{array}$ & $3.40 \times 10^{-4}$ & $8.70 \times 10^{-4}$ & $4.39 \times 10^{-4}$ \\
\hline $10^{-6}$ & $\begin{array}{c}3.20 \times 10^{-3} \\
0.53125\end{array}$ & $\begin{array}{c}1.70 \times 10^{-3} \\
0.211765\end{array}$ & $3.60 \times 10^{-4}$ & $8.93 \times 10^{-4}$ & $4.48 \times 10^{-4}$ \\
\hline $10^{-8}$ & $\begin{array}{c}3.40 \times 10^{-3} \\
0.50\end{array}$ & $\begin{array}{c}1.70 \times 10^{-3} \\
0.211765\end{array}$ & $3.60 \times 10^{-4}$ & $8.94 \times 10^{-4}$ & $4.49 \times 10^{-4}$ \\
\hline
\end{tabular}




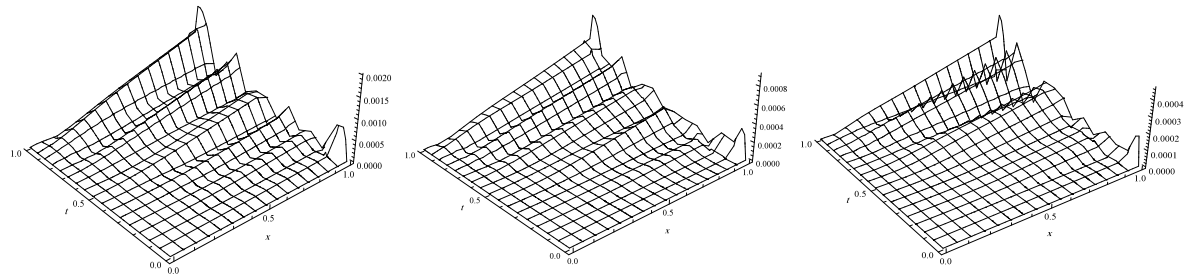

Figure 1. Max absolute error with $\varepsilon=10^{-8}$ (Left: $N=200$; Middle: $N=400$; Right: $N=800)$ for Example 1 .
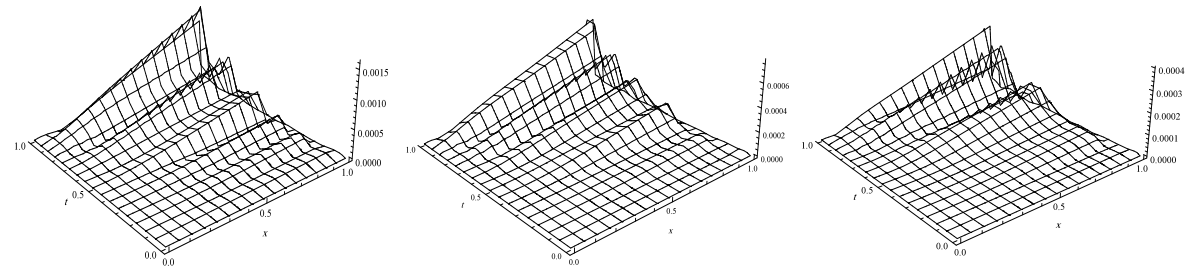

Figure 2. Max absolute error with $\varepsilon=10^{-6}$ and $n=10$ (Left: $N=200$; Middle: $N=400$; Right: $N=800)$ for Example 2 .
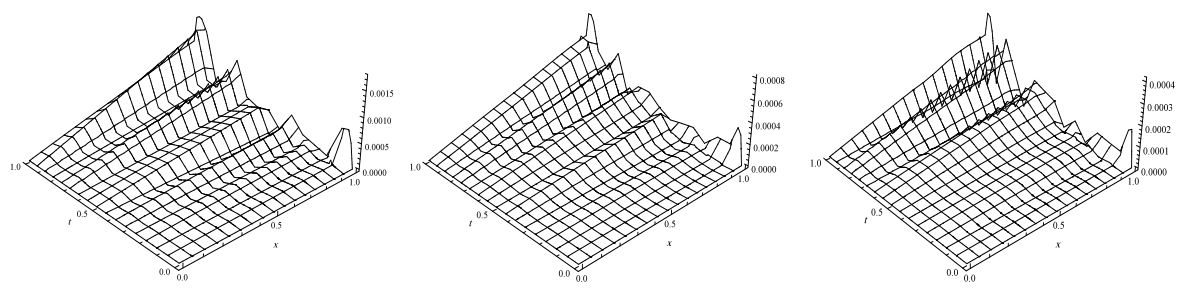

Figure 3. Max absolute error with $\varepsilon=10^{-8}$ and $n=10$ (Left: $N=200$; Middle: $N=400$; Right: $N=800$ ) for Example 3 .

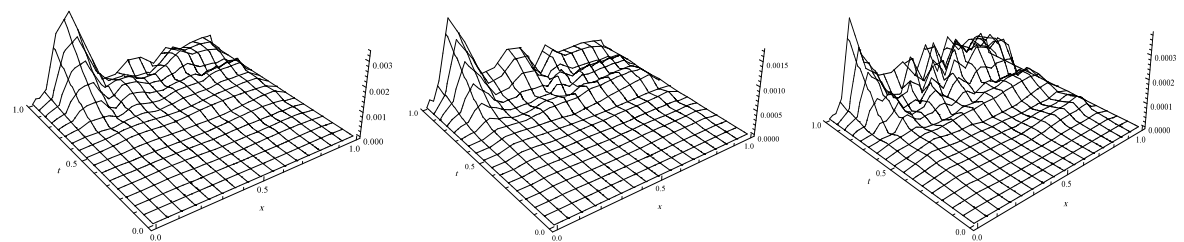

Figure 4. Max error with $\varepsilon=10^{-6}$ (Left: $N=200$; Middle: $N=400$; Right: $N=800$ ) for Example 4. 
Table 2. Max absolute error $\left(\mathbb{E}^{\mathcal{Y}_{N, n}(x, t)}=\operatorname{Max}\left|\mathcal{Y}_{N, n}(x, t)-y(x, t)\right|,(x, t) \in D\right)$ with

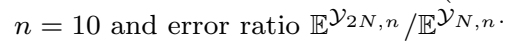

\begin{tabular}{|c|c|c|c|c|c|}
\hline \multirow[b]{2}{*}{$N$} & \multicolumn{3}{|l|}{ PM } & \multicolumn{2}{|l|}{$[17]$} \\
\hline & 200 & 400 & 800 & $512 \times 160$ & $1024 \times 320$ \\
\hline
\end{tabular}

Ex.1

$\begin{array}{cccccc}10^{0} & 1.50 \times 10^{-4} & 5.50 \times 10^{-5} & 2.30 \times 10^{-5} & 5.41 \times 10^{-5} & 2.67 \times 10^{-5} \\ & 0.366667 & 0.418182 & & & \\ 10^{-2} & 3.20 \times 10^{-3} & 1.30 \times 10^{-3} & 5.50 \times 10^{-4} & 4.55 \times 10^{-3} & 2.52 \times 10^{-3} \\ & 0.40625 & 0.423077 & & & \\ 10^{-4} & 1.60 \times 10^{-3} & 8.00 \times 10^{-4} & 4.00 \times 10^{-4} & 5.42 \times 10^{-3} & 2.74 \times 10^{-3} \\ & 0.50 & 0.50 & & & \\ 10^{-6} & 1.60 \times 10^{-3} & 8.00 \times 10^{-4} & 4.00 \times 10^{-4} & 5.52 \times 10^{-3} & 2.76 \times 10^{-3} \\ & 0.50 & 0.50 & & & \\ 10^{-8} & 2.00 \times 10^{-3} & 8.50 \times 10^{-4} & 4.40 \times 10^{-4} & 5.55 \times 10^{-3} & 2.77 \times 10^{-3} \\ & 0.425 & 0.517647 & & & \end{array}$

Ex.2

\begin{tabular}{|c|c|c|c|c|}
\hline $\begin{array}{c}1.50 \times 10^{-4} \\
0.333333\end{array}$ & $\begin{array}{c}5.00 \times 10^{-5} \\
0.440\end{array}$ & $2.20 \times 10^{-5}$ & $4.53 \times 10^{-5}$ & $2.28 \times 10^{-5}$ \\
\hline $\begin{array}{c}2.60 \times 10^{-3} \\
0.423077\end{array}$ & $\begin{array}{c}1.10 \times 10^{-3} \\
0.418182\end{array}$ & $4.60 \times 10^{-4}$ & $2.83 \times 10^{-3}$ & $1.42 \times 10^{-3}$ \\
\hline $\begin{array}{c}1.60 \times 10^{-3} \\
0.46875\end{array}$ & $\begin{array}{c}7.50 \times 10^{-4} \\
0.533333\end{array}$ & $4.00 \times 10^{-4}$ & $3.84 \times 10^{-3}$ & $1.93 \times 10^{-3}$ \\
\hline $\begin{array}{c}1.60 \times 10^{-3} \\
0.46875\end{array}$ & $\begin{array}{c}7.50 \times 10^{-4} \\
0.533333\end{array}$ & $4.00 \times 10^{-4}$ & $3.92 \times 10^{-3}$ & $1.96 \times 10^{-3}$ \\
\hline $\begin{array}{c}1.80 \times 10^{-3} \\
0.472222\end{array}$ & $\begin{array}{c}8.50 \times 10^{-4} \\
0.517647\end{array}$ & $4.40 \times 10^{-4}$ & $3.95 \times 10^{-3}$ & $1.96 \times 10^{-3}$ \\
\hline & & & $N=256 \times 80$ & $N=512 \times 160$ \\
\hline $\begin{array}{c}1.50 \times 10^{-4} \\
0.366667\end{array}$ & $\begin{array}{c}5.50 \times 10^{-5} \\
0.40\end{array}$ & $2.20 \times 10^{-5}$ & $2.83 \times 10^{-5}$ & $1.42 \times 10^{-5}$ \\
\hline $\begin{array}{c}2.60 \times 10^{-3} \\
0.423077\end{array}$ & $\begin{array}{c}1.10 \times 10^{-3} \\
0.418182\end{array}$ & $4.60 \times 10^{-4}$ & $5.21 \times 10^{-3}$ & $2.70 \times 10^{-3}$ \\
\hline $\begin{array}{c}1.60 \times 10^{-3} \\
0.46875\end{array}$ & $\begin{array}{c}7.50 \times 10^{-4} \\
0.533333\end{array}$ & $4.00 \times 10^{-4}$ & $8.28 \times 10^{-3}$ & $4.17 \times 10^{-3}$ \\
\hline $\begin{array}{c}1.60 \times 10^{-3} \\
0.46875\end{array}$ & $\begin{array}{c}7.50 \times 10^{-4} \\
0.506667\end{array}$ & $3.80 \times 10^{-4}$ & $8.85 \times 10^{-3}$ & $4.28 \times 10^{-3}$ \\
\hline $\begin{array}{c}1.70 \times 10^{-3} \\
0.470588\end{array}$ & $\begin{array}{c}8.00 \times 10^{-4} \\
0.525\end{array}$ & $4.20 \times 10^{-4}$ & $8.65 \times 10^{-3}$ & $4.30 \times 10^{-3}$ \\
\hline
\end{tabular}


Table 3. Max absolute errors for derivative of the approximate solutions in Example 3.

\begin{tabular}{cccc}
\hline & Present Method & \\
\cline { 2 - 4 } & $N=200$ & $N=400$ & $N=800$ \\
\hline$\varepsilon=10^{-6}$ & & & \\
$\mathbb{E}^{\partial_{t} \mathcal{Y}_{N, n}(x, t)}$ & $1.90 \times 10^{-2}$ & $1.20 \times 10^{-2}$ & $8.50 \times 10^{-3}$ \\
$\mathbb{E}^{\partial_{x} \mathcal{Y}_{N, n}(x, t)}$ & $2.00 \times 10^{13}$ & $2.00 \times 10^{14}$ & $6.00 \times 10^{13}$ \\
& & & \\
$\varepsilon=10^{-8}$ & & & \\
$\mathbb{E}^{\partial_{t} \mathcal{Y}_{N, n}(x, t)}$ & $3.40 \times 10^{-2}$ & $2.30 \times 10^{-2}$ & $1.60 \times 10^{-2}$ \\
$\mathbb{E}^{\partial_{x} \mathcal{Y}_{N, n}(x, t)}$ & $8.00 \times 10^{22}$ & $8.50 \times 10^{22}$ & $2.00 \times 10^{23}$ \\
& & & \\
\hline
\end{tabular}
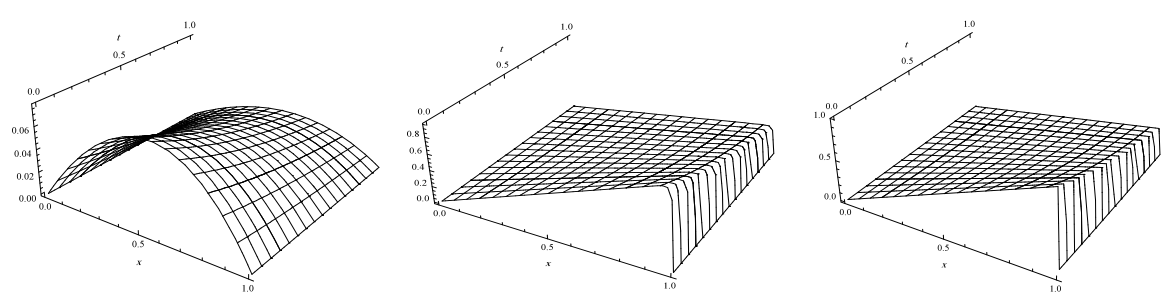

Figure 5. Approximate solution with $N=200$ and $n=10$ (Left: $\varepsilon=1$; Middle: $\varepsilon=10^{-2}$; Right: $\varepsilon=10^{-6}$ ) for Examples 1, 2, 3 .
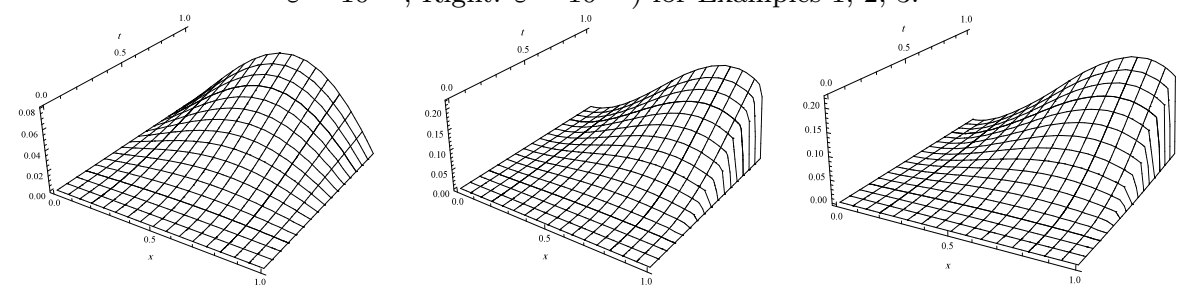

Figure 6. Approximate solution with $N=200$ (Left: $\varepsilon=1$; Middle: $\varepsilon=10^{-2}$; Right: $\varepsilon=10^{-6}$ ) for Example 4 .
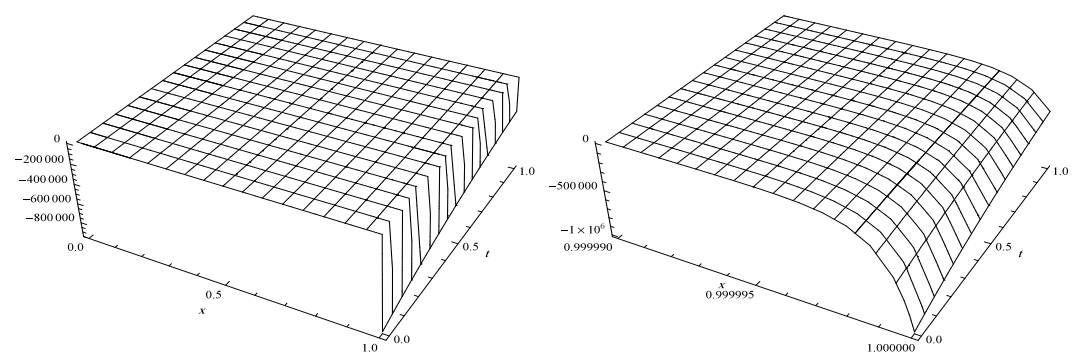

Figure 7. Derivative of the solution relative to $x\left(\partial_{x} y(x, t)\right)$ throughout region $D$ for Examples 1, 2, 3, with $\varepsilon=10^{-6}$ (Left: derivative of the solution; Right: boundary layer behavior). 